Research Article

\title{
Identification and Validation of Core Genes Involved in the Development of Papillary Thyroid Carcinoma via Bioinformatics Analysis
}

\author{
Xiaoyan Li, ${ }^{1}$ Jing He, ${ }^{1}$ Mingxia Zhou $\mathbb{D}^{2},{ }^{2}$ Yun Cao, ${ }^{1}$ Yiting Jin $\mathbb{D},{ }^{1}$ and Qiang Zou $\mathbb{D}^{1}$ \\ ${ }^{1}$ Department of General Surgery, Huashan Hospital, Fudan University, Shanghai, China \\ ${ }^{2}$ Department of Gastroenterology, Xinhua Hospital, School of Medicine, Shanghai Jiao Tong University, Shanghai, China \\ Correspondence should be addressed to Yiting Jin; clara_raky@aliyun.com and Qiang Zou; zouqiang_hs@163.com
}

Received 25 March 2019; Accepted 20 June 2019; Published 8 September 2019

Academic Editor: Byung-Hoon Jeong

Copyright (C) 2019 Xiaoyan Li et al. This is an open access article distributed under the Creative Commons Attribution License, which permits unrestricted use, distribution, and reproduction in any medium, provided the original work is properly cited.

\begin{abstract}
Background. Papillary thyroid carcinoma (PTC) is a common endocrine malignant neoplasm, and its incidence increases continuously worldwide in the recent years. However, efficient clinical biomarkers were still deficient; the present research is aimed at exploring significant core genes of PTC. Methods. We integrated three cohorts to identify hub genes and pathways associated with PTC by comprehensive bioinformatics analysis. Expression profiles GSE33630, GSE35570, and GSE60542, including 114 PTC tissues and 126 normal tissues, were enrolled in this research. Gene ontology (GO) and Kyoto Encyclopedia of Genes and Genomes (KEGG) pathway enrichment analyses were utilized to search for the crucial biological behaviors and pathways involved in PTC carcinogenesis. Protein-protein interaction (PPI) network was constructed, and significant modules were deeply studied. Results. A total of 831 differentially expressed genes (DEGs) were discovered, comprising 410 upregulated and 421 downregulated genes in PTC tissues compared to normal thyroid tissues. PPI network analysis demonstrated the interactions between those DEGs, and top 10 pivotal genes (TGFB1, CXCL8, LRRK2, CD44, CCND1, JUN, DCN, BCL2, ACACB, and CXCL12) with highest degree of connectivity were extracted from the network and verified by TCGA dataset and RT-PCR experiment of PTC samples. Four of the hub genes (CXCL8, DCN, BCL2, and ACACB) were linked to the prognosis of PTC patients and considered as clinically relevant core genes via survival analysis. Conclusion. In conclusion, we propose a series of key genes associated with PTC development and these genes could serve as the diagnostic biomarkers or therapeutic targets in the future treatment for PTC.
\end{abstract}

\section{Introduction}

Thyroid cancer accounts for $96 \%$ malignant tumors of the head and neck, which is the most common malignant tumor in endocrine organs and the fifth most common cancer in women worldwide $[1,2]$. The incidence of thyroid cancer continues to increase during the past 30 years, which has the fastest growth rate among all solid malignant tumors, generating a serious impact on the quality of life and huge social economic burden for patients [3]. The 2017 edition of American cancer statistics indicated that the incidence of thyroid cancer accounted for $3.4 \%$ of all malignant tumors.
According to an analysis of thyroid cancer data from the World Health Organization during 1960 to 2012, the incidence of thyroid cancer in most countries and regions around the world presents a rapid growth in the last half century [4]. Thyroid cancer can be classified into four subtypes: papillary thyroid carcinoma (PTC), follicular thyroid carcinoma (FTC), medullary thyroid carcinoma (MTC), and anaplastic thyroid carcinoma (ATC), among which PTC is the most common type, accounting for about $80 \%$ of all thyroid cancer. Most of the patients with differentiated thyroid cancer (DTC) can obtain satisfactory therapeutic effects through surgical excision and radioactive iodine therapy 
and an overall 10-year survival rate exceeding 90\% [5, 6]. However, a portion of thyroid cancer patients develop distant metastases and exhibit poor prognosis [7].

At present, the cause of PTC is not very clear. Several studies reported that the incidence of thyroid cancer is influenced by many factors, such as sex, age, region, and ethnic differences $[8,9]$. Many studies have reported that the pathogenesis of PTC is related to BRAF mutation and RET/PTC and PAX8/PPAR rearrangement; one hypothesis speculated that the activation of serine/threonine kinase caused by mutations of BRAF at T1799A and V599E may be an alternative mechanism of oncogenic mitogen-activated protein kinase (MAPK) pathway activation [10-12], and other groups found that RET/PTC rearrangement promotes the proliferation and migration of PTC cells by regulating nuclear factor$\kappa \mathrm{B}(\mathrm{NF}-\kappa \mathrm{B})$ activity and relative oncogenic protein expression [13-15]. PAX8/PPAR rearrangement was also involved in thyroid tumorigenesis. PAX8 regulates thyroglobulin, thyroid peroxidase, and thyrotropin receptor gene promoters; it also regulates the differentiation and growth of thyroid cells [16]. PPAR $\gamma$ is one of the nuclear receptors, which participates in inflammation, cell cycle control, apoptosis, and oncogenesis. PAX/PPAR rearrangement intervenes the transcription pathway, upregulates relative gene promoter activity, and finally promotes PTC progression [17-19]. However, these theories still cannot explain the pathogenesis of all PTCs. Therefore, further studies of the molecular mechanisms of PTC and seeking novel biomarkers for PTC seem to be of great significance and clinical value.

With wide application of high-throughput sequencing and public databases, the bioinformatics analysis has been broadly used as a powerful tool for cancer diagnosis, classification, and prognosis prediction, especially in obtaining gene general alterations during tumorigenesis. In this study, three original microarray datasets GSE33630, GSE35570, and GSE60542 were selected from Gene Expression Omnibus (GEO, https://www.ncbi.nlm.nih.gov/geo/) with a total of 114 PTC samples and 126 normal thyroid tissues, which owns the largest sample size compared with a previously published study with a total of 92 PTC samples and 79 normal thyroid tissue samples [20]. With the adhibition of the GEO2R online tool, 831 DEGs (differentially expressed genes) were detected: 410 of those genes were upregulated and 421 were downregulated. Based on those DEGs, the functional roles and related pathways were jointly performed by Database for Annotation, Visualization, and Integrated Discovery (DAVID) and other online tools. In addition, we established the PPI network of the DEGs and ten hub genes (TGFB1, JUN, CXCL8, LRRK2, CD44, DCN, BCL2, ACACB, CCND1, and CXCL12) were identified and verified by RTPCR experiments in our own thyroid samples in Huashan Hospital. These findings may provide us with a better understanding of the molecular mechanism and possible treatment targets for PTC.

\section{Materials and Methods}

2.1. Gene Expression Profile Data. The gene expression profile datasets GSE33630, GSE35570, and GSE60542 were down-
TABLE 1: Sequences of primers for RT-PCR.

\begin{tabular}{|c|c|}
\hline Gene & Primer sequences \\
\hline \multirow{2}{*}{ TGFB1 } & Forward: $5^{\prime}$-CAATTCCTGGCGATACCTCAG-3' \\
\hline & Reverse: 5'-GCACAACTCCGGTGACATCAA-3' \\
\hline \multirow{2}{*}{$J U N$} & Forward: $5^{\prime}$-TCCAAGTGCCGAAAAAGGAAG-3' \\
\hline & Reverse: 5'-CGAGTTCTGAGCTTTCAAGGT-3' \\
\hline \multirow{2}{*}{ CXCL8 } & Forward: 5' -ACTGAGAGTGATTGAGAGTGGAC-3' \\
\hline & Reverse: 5' -AACCCTCTGCACCCAGTTTTC-3' \\
\hline \multirow{2}{*}{$L R R K 2$} & Forward: 5' -ATGAGTGGCAATGTCAGGTGT-3' \\
\hline & Reverse: 5'-AATGTAAGCCTATGGAGCAAACA-3' \\
\hline \multirow{2}{*}{ CD44 } & Forward: 5'-CTGCCGCTTTGCAGGTGTA-3' \\
\hline & Reverse: 5'-CATTGTGGGCAAGGTGCTATT-3' \\
\hline \multirow{2}{*}{$D C N$} & Forward: $5^{\prime}$-ATGAAGGCCACTATCATCCTCC-3' \\
\hline & Reverse: 5'-GTCGCGGTCATCAGGAACTT-3' \\
\hline \multirow{2}{*}{$B c l-2$} & Forward: 5'-GGTGGGGTCATGTGTGTGG-3' \\
\hline & Reverse: 5' -CGGTTCAGGTACTCAGTCATCC-3’ \\
\hline \multirow{2}{*}{$A C A C B$} & Forward: $5^{\prime}$-CAAGCCGATCACCAAGAGTAAA-3' \\
\hline & Reverse: 5' -CCCTGAGTTATCAGAGGCTGG-3' \\
\hline \multirow{2}{*}{ CCND1 } & Forward: 5' -CAATGACCCCGCACGATTTC-3' \\
\hline & Reverse: 5' -CATGGAGGGCGGATTGGAA-3' \\
\hline \multirow{2}{*}{ CXCL12 } & Forward: 5' -CCATGCCGATTCTTCGAAAG-3' \\
\hline & Reverse: $5^{\prime}$-TTCAGCCGGGCTACAATCTG-3' \\
\hline \multirow{2}{*}{ GAPDH } & Forward: $5^{\prime}$-GCACCGTCAAGGCTGAGAAC-3' \\
\hline & Reverse: 5'-TGGTGAAGACGCCAGTGGA-3' \\
\hline
\end{tabular}

loaded from GEO database. A platform of these three GEO datasets was GPL570 (Affymetrix Human Genome U133 Plus 2.0 Array). The GSE33630 dataset included 94 samples derived from PTC (papillary thyroid cancer) patients (49 tumor tissues and 45 nontumoral thyroid tissues). In the GSE35570 dataset, there consists of 32 PTC tissues and 51 normal samples. As for the GSE60542 dataset, 63 samples were included with 33 tumor tissues and 30 noncancerous tissues. We chose these three datasets for integrated analysis in this study because all of them had a pretty large sample size compared to other datasets about PTC in GEO data repository.

2.2. DEG Identification. A GEO2R (https://www.ncbi.nlm. nih.gov/geo/geo2r/) online program was applied to detect differentially expressed genes between PTC tumor and nontumor tissues [21]. $\left|\log _{2} \mathrm{FC}\right|>1$ and the adjusted $p$ value $<0.05$ were set as cutoff criteria. Then, a total of 831 DEGs were identified, containing 410 upregulated genes and 421 downregulated genes, and we selected the top ten genes with highest degree of connectivity as hub genes. For the validation of the expression of these hub genes, the data from GEPIA was extracted and analyzed. The TCGA (The 


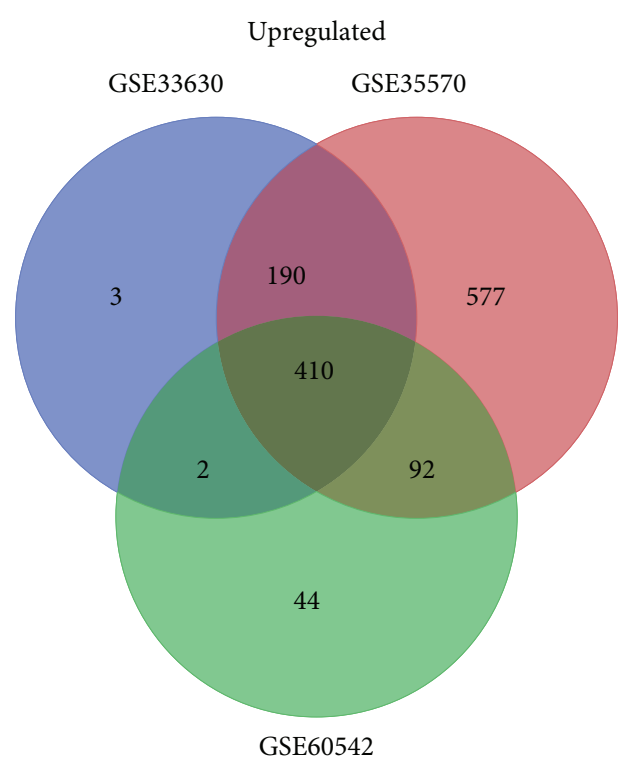

(a)

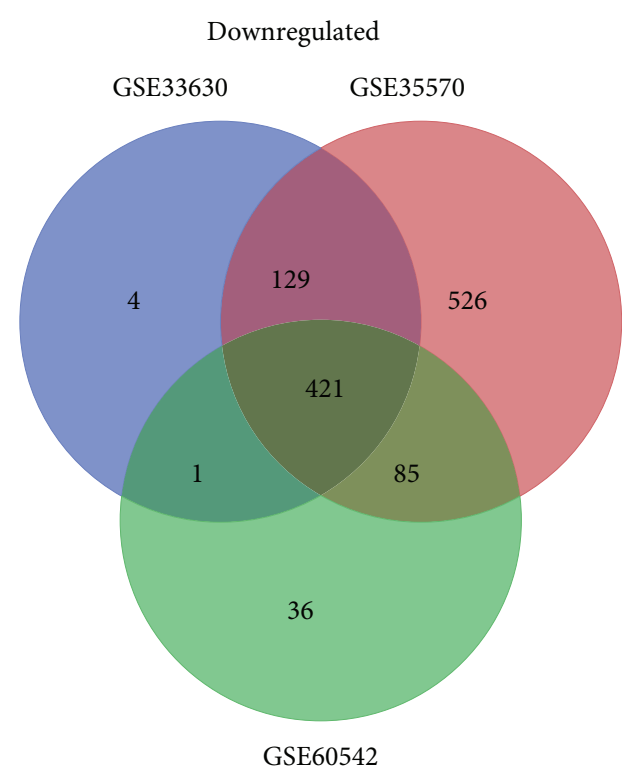

(b)

FIGURE 1: Identification of aberrantly expressed genes in gene expression profile datasets GSE33630, GSE35570, and GSE60542. Venn diagram of upregulated genes (a) and downregulated genes (b).

Cancer Genome Atlas) PTC data included 337 normal and 512 PTCs in the GEPIA. The GEPIA (http://gepia.cancerpku.cn/) is a newly developed web-based tool to provide differential expression analysis about numerous kinds of cancer based on the data from TCGA and GTEx (Genotype-Tissue Expression) [22].

2.3. Functional Network Establishment of DEGs. To annotate the upregulated and downregulated DEGs presented from our analysis on the functional level, we discovered the gene ontology (GO) functional enrichment term including biological process (BP), cellular component (CC), molecular function (MF), and vital pathways using the online application DAVID (version 6.8, https://david.ncifcrf.gov/), Reactome (http://www.reactome.org), and clusterProfiler package in $\mathrm{R}$ (http://www.bioconductor.org/packages/release/bioc/html/ clusterProfiler.html), with the threshold set as $p<0.05$ [23].

2.4. PPI Network and Module Analysis. To determine the relationship between upregulated and downregulated DEGs, we uploaded all DEGs to the STRING database (version 10.5, https://string-db.org/cgi/input.pl), choosing a combined score $>0.4$ to construct PPI. Then, the Cytoscape software (version 3.6.0, http://www.cytoscape.org/) was utilized to construct the network of PPI. The Molecular Complex Detection (MCODE) app in Cytoscape was recruited to analyze modules of the PPI network. The KEGG pathway analysis of genes in each module was performed by DAVID.

2.5. Tissue Samples. 32 pairs of primary human PTC cancerous tissues and their corresponding adjacent normal thyroid tissues were obtained from Huashan Hospital of Fudan University. Samples were obtained and snap frozen at liquid nitrogen immediately and stored at $-80^{\circ} \mathrm{C}$ until analysis. All
PTC specimens were confirmed by histopathology. Ethical approval was obtained from the ethics committee of Huashan Hospital, Fudan University, and written informed consent was obtained from each patient.

2.6. RNA Extraction and RT-PCR. Total RNA was isolated from PTC cells and tissues using the TRIzol reagent (Invitrogen, USA). cDNA was synthesized using $2.0 \mu \mathrm{g}$ of total RNA with the PrimeScript ${ }^{\mathrm{TM}}$ RT reagent kit (TaKaRa Biotechnology, Japan). The SYBR-Green Supermix kit (TaKaRa) was employed to detect the relative mRNA expression in the ABI 7900 instrument (Applied Biosystems Inc.). The relative expression levels were determined by the $2^{-\Delta \Delta \mathrm{Ct}}$ method and normalized to internal control GAPDH [24, 25]. All qPCR reactions were performed in triplicate. The primers used to explore mRNA expression of ten hub genes were shown in Table 1.

2.7. Statistical Analysis. The expression of related genes was presented as mean \pm SD and analyzed using the paired $t$-test. All data were analyzed with GraphPad Prism 7.0, and $p<0.05$ was considered as a significant difference.

\section{Results}

3.1. Screening of DEGs between PTC and Normal Thyroid Tissues. Three GEO datasets GSE33630, GSE35570, and GSE60542, a total of 114 PTC tissues and 126 normal thyroid tissues, were analyzed in our research. According to the cutoff criteria: $\left|\log _{2} \mathrm{FC}\right|>1$ and the adjusted $p$ value $<0.05$, we extracted 605, 1269, and 548 upregulated DEGs and 555, 1161, and 543 downregulated DEGs from the expression profile datasets GSE33630, GSE35570, and GSE60542, respectively (Figure 1). 410 upregulated DEGs and 421 
TABLE 2: Gene ontology analysis of differentially expressed genes in PTC.

\begin{tabular}{|c|c|c|c|c|c|}
\hline Expression & Category & ID & Term & Count & $p$ value \\
\hline \multirow{15}{*}{ Downregulated } & GOTERM_BP_FAT & GO:0007167 & Enzyme-linked receptor protein signaling pathway & 47 & $3.40 E-08$ \\
\hline & GOTERM_BP_FAT & GO:0007399 & Nervous system development & 80 & $8.50 E-08$ \\
\hline & GOTERM_BP_FAT & GO:0061061 & Muscle structure development & 34 & $1.20 E-07$ \\
\hline & GOTERM_BP_FAT & GO:0010517 & Regulation of phospholipase activity & 11 & $4.20 E-07$ \\
\hline & GOTERM_BP_FAT & GO:0040007 & Growth & 43 & $8.80 E-07$ \\
\hline & GOTERM_CC_FAT & GO:0005578 & Proteinaceous extracellular matrix & 31 & $1.50 E-10$ \\
\hline & GOTERM_CC_FAT & GO:0031012 & Extracellular matrix & 36 & $2.20 E-09$ \\
\hline & GOTERM_CC_FAT & GO:0044421 & Extracellular region part & 124 & $1.80 E-07$ \\
\hline & GOTERM_CC_FAT & GO:0005576 & Extracellular region & 137 & $2.50 E-06$ \\
\hline & GOTERM_CC_FAT & GO:0030018 & $\mathrm{Z}$ disc & 12 & $4.00 E-05$ \\
\hline & GOTERM_MF_FAT & GO:1901681 & Sulfur compound binding & 17 & $1.90 E-05$ \\
\hline & GOTERM_MF_FAT & GO:0005539 & Glycosaminoglycan binding & 16 & $2.00 E-05$ \\
\hline & GOTERM_MF_FAT & GO:0008201 & Heparin binding & 13 & $9.20 E-05$ \\
\hline & GOTERM_MF_FAT & GO:0005102 & Receptor binding & 49 & $4.30 E-04$ \\
\hline & GOTERM_MF_FAT & GO:0019199 & Transmembrane receptor protein kinase activity & 8 & $1.30 E-03$ \\
\hline \multirow{15}{*}{ Upregulated } & GOTERM_BP_FAT & GO:0030198 & Extracellular matrix organization & 35 & $2.10 E-14$ \\
\hline & GOTERM_BP_FAT & GO:0007155 & Cell adhesion & 85 & $2.90 E-14$ \\
\hline & GOTERM_BP_FAT & GO:2000026 & Regulation of multicellular organismal development & 78 & $6.70 E-11$ \\
\hline & GOTERM_BP_FAT & GO:0040011 & Locomotion & 70 & $6.10 E-10$ \\
\hline & GOTERM_BP_FAT & GO:0051094 & Positive regulation of developmental process & 56 & $8.80 E-10$ \\
\hline & GOTERM_CC_FAT & GO:0005576 & Extracellular region & 160 & $2.20 E-10$ \\
\hline & GOTERM_CC_FAT & GO:0044421 & Extracellular region part & 141 & $2.40 E-10$ \\
\hline & GOTERM_CC_FAT & GO:0031982 & Membrane-bounded vesicle & 115 & $3.80 E-05$ \\
\hline & GOTERM_CC_FAT & GO:0070062 & Extracellular exosome & 94 & $4.90 E-05$ \\
\hline & GOTERM_CC_FAT & GO:1903561 & Extracellular vesicle & 94 & $6.00 E-05$ \\
\hline & GOTERM_MF_FAT & GO:0005539 & Glycosaminoglycan binding & 20 & $7.60 E-08$ \\
\hline & GOTERM_MF_FAT & GO:0005102 & Receptor binding & 60 & $5.40 E-07$ \\
\hline & GOTERM_MF_FAT & GO:0050840 & Extracellular matrix binding & 10 & $1.30 E-06$ \\
\hline & GOTERM_MF_FAT & GO:0042802 & Identical protein binding & 54 & $6.00 E-06$ \\
\hline & GOTERM_MF_FAT & GO:0098772 & Molecular function regulator & 52 & $5.60 E-05$ \\
\hline
\end{tabular}

Abbreviations: GO: gene ontology; BP: biological process; MF: molecular function; CC: cellular component.

downregulated DEGs were consistently presented in all three datasets and were used for further study.

3.2. Gene Ontology Functional Analysis. In order to obtain a more in-depth understanding of the selected DEGs, GO function was performed using DAVID version 6.8 and the clusterProfiler package in $\mathrm{R}$ statistical software (version 3.4.2). As shown in Table 2 and Figure 2(a), the upregulated DEGs mainly enriched in biological process (BP) terms were associated with extracellular matrix organization, cell adhesion, and regulation of protein serine/threonine kinase activity. Meanwhile, the downregulated DEGs were linked to nervous system development, muscle tissue development, and regulation of phospholipase activity (Figure 2(b)). As for the cellular component (CC), the upregulated DEGs were enriched in the proteinaceous extracellular region, endoplasmic reticulum lumen, and membrane-bounded vesicle, while the downregulated DEGs were related to the proteinaceous extracellular matrix. In the molecular func- tion (MF) group, it was concluded that the upregulated DEGs were mainly involved in receptor binding and glycosaminoglycan binding and the downregulated DEGs were closely related to sulfur compound binding and nucleosidetriphosphatase regulator activity.

3.3. KEGG Signaling Pathway Enrichment Analysis. To systematically clarify the key pathways involved in PTC pathogenesis, pathway enrichment analysis was carried out using online websites of the KEGG pathway, Reactome, and the clusterProfiler R package. The pathways enriched by upregulated DEGs were mainly related to ECM-receptor interaction, focal adhesion, and p53 signaling pathway. However, the central pathways shown in downregulated DEGs were mineral absorption, thyroid hormone synthesis, and TGFbeta signaling pathway (Table 3 and Figure 3).

3.4. PPI Network Construction and Modular Analysis. The STRING online database was employed to construct the 


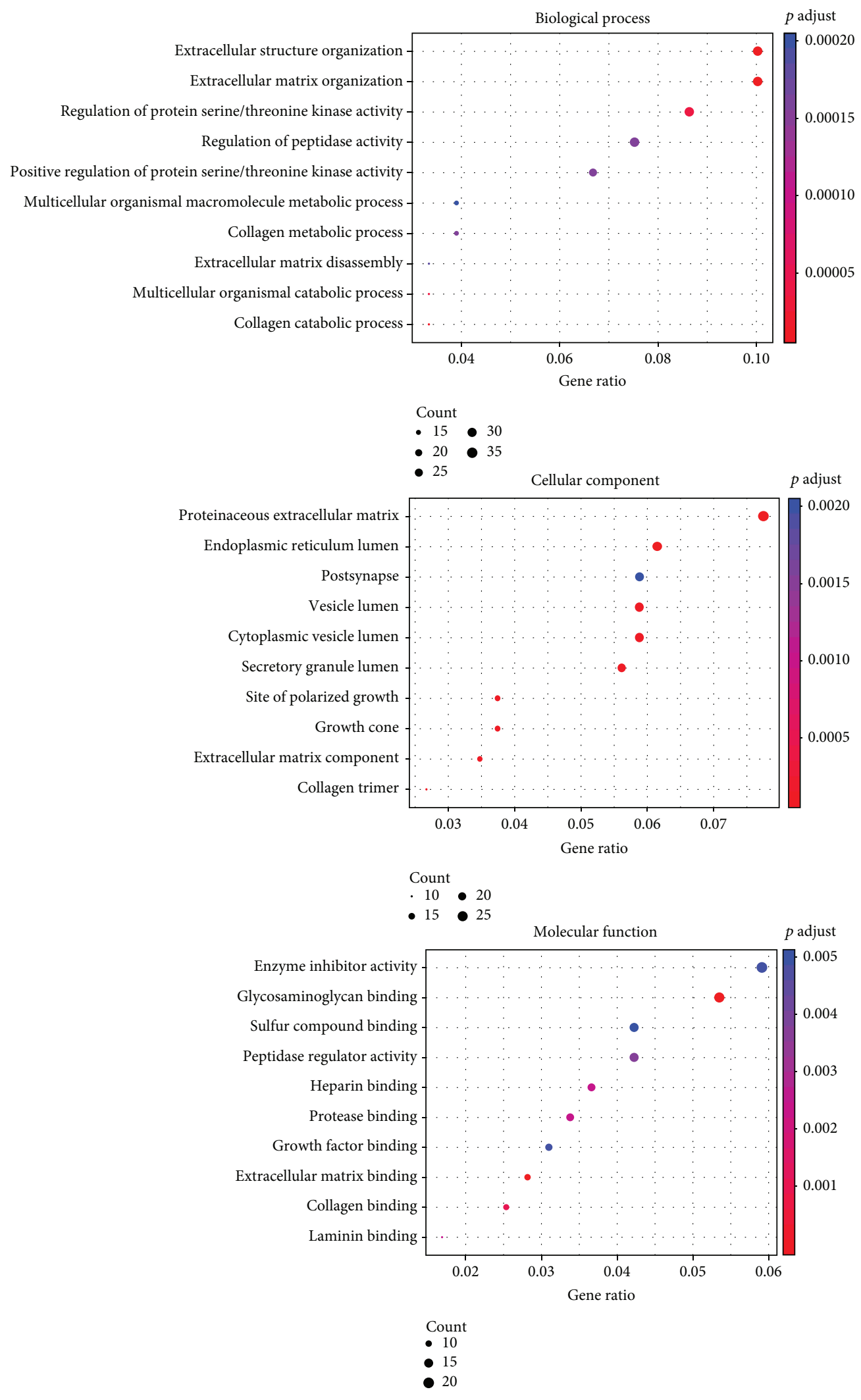

(a)

Figure 2: Continued. 


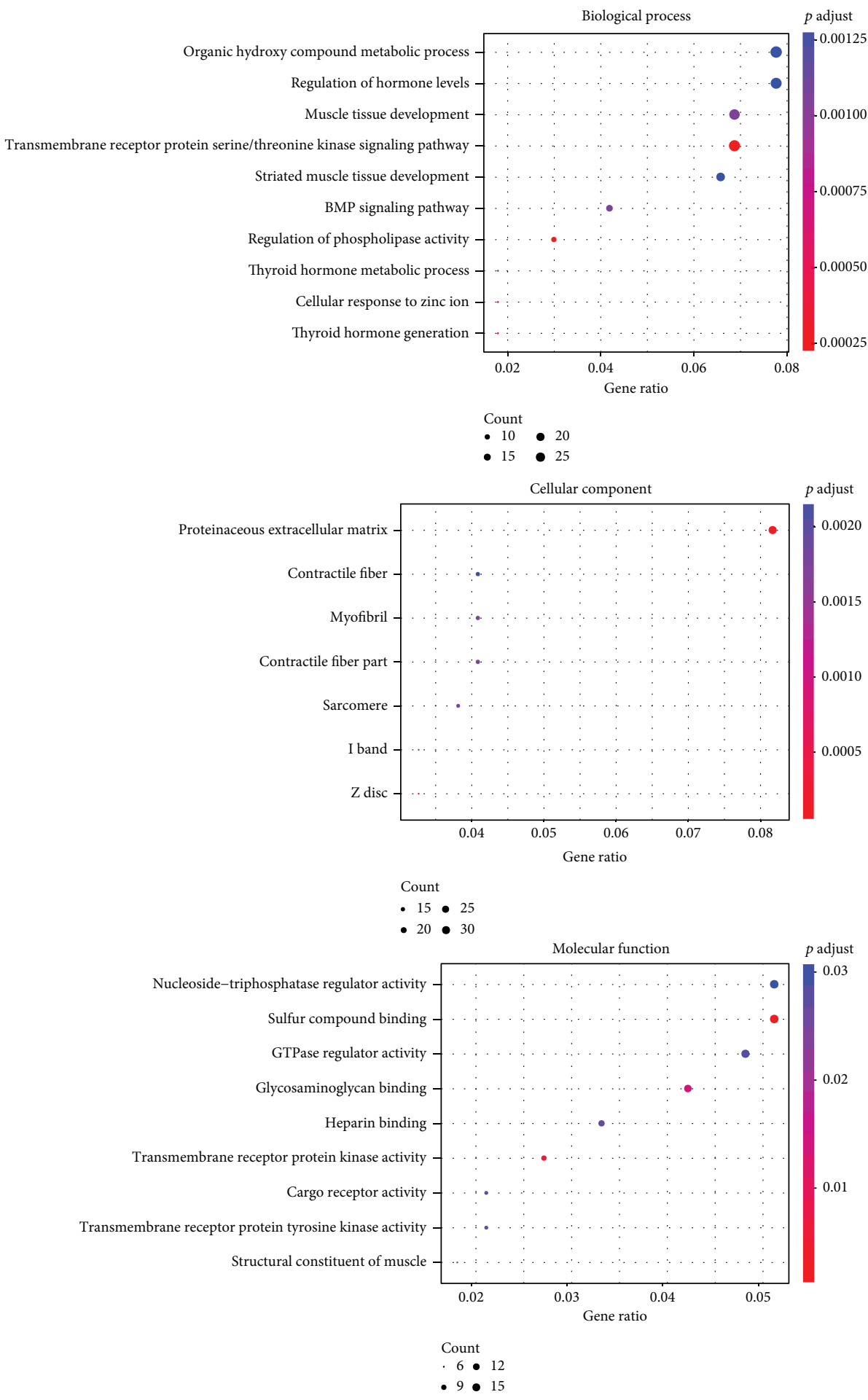

(b)

FIGURE 2: The enriched gene ontology categories including biological process, cellular component, and molecular function of DEGs. (a) The GO functional enrichment analysis of upregulated DEGs. (b) The GO functional enrichment analysis of downregulated DEGs based on clusterProfiler. Abbreviations: DEGs: differentially expressed genes; GO: gene ontology.

PPI network; then, the Cytoscape software was used to visualize the protein interactions. Considering degree $>10$ as the cutoff criterion, a total of 108 DEGs (including 62 upregulated DEGs and 46 downregulated DEGs) in the 831 com- monly altered DEGs were filtered to establish the PPI network, which comprises 108 nodes and 769 edges (Figure 4). The PPI network provides us with the most significant ten nodes (TGFB1, CXCL8, LRRK2, CD44, CCND1, 
TABLE 3: KEGG and Reactome pathway enrichment analysis of differentially expressed genes in PTC.

\begin{tabular}{|c|c|c|c|c|c|}
\hline Expression & Category & ID & Term & Count & $p$ value \\
\hline \multirow{5}{*}{ Downregulated } & KEGG_PATHWAY & hsa04978 & Mineral absorption & 6 & $2.90 E-03$ \\
\hline & KEGG_PATHWAY & hsa04918 & Thyroid hormone synthesis & 7 & $3.80 E-03$ \\
\hline & KEGG_PATHWAY & hsa04350 & TGF-beta signaling pathway & 6 & $3.50 E-02$ \\
\hline & KEGG_PATHWAY & hsa00350 & Tyrosine metabolism & 4 & $3.90 E-02$ \\
\hline & KEGG_PATHWAY & hsa04020 & Calcium signaling pathway & 9 & $3.90 E-02$ \\
\hline \multirow{5}{*}{ Upregulated } & KEGG_PATHWAY & hsa04512 & ECM-receptor interaction & 15 & $1.10 E-08$ \\
\hline & KEGG_PATHWAY & hsa04510 & Focal adhesion & 17 & $2.50 E-05$ \\
\hline & KEGG_PATHWAY & hsa04115 & p53 signaling pathway & 9 & $1.60 E-04$ \\
\hline & KEGG_PATHWAY & hsa05205 & Proteoglycans in cancer & 15 & $2.50 E-04$ \\
\hline & KEGG_PATHWAY & hsa04151 & PI3K-Akt signaling pathway & 17 & $7.20 E-03$ \\
\hline \multirow{5}{*}{ Downregulated } & REACTOME_PATHWAY & R-HSA-5661231 & Metallothioneins bind metals & 6 & $2.33 E-05$ \\
\hline & REACTOME_PATHWAY & R-HSA-5660526 & Response to metal ions & 6 & $1.03 E-04$ \\
\hline & REACTOME_PATHWAY & R-HSA-3560782 & Diseases associated with glycosaminoglycan metabolism & 7 & 0.001726 \\
\hline & REACTOME_PATHWAY & R-HSA-8851708 & Signaling by FGFR2 IIIa TM & 5 & 0.001887 \\
\hline & REACTOME_PATHWAY & R-HSA-2129379 & Molecules associated with elastic fibres & 6 & 0.002228 \\
\hline \multirow{5}{*}{ Upregulated } & REACTOME_PATHWAY & R-HSA-1474244 & Extracellular matrix organization & 35 & $1.39 E-08$ \\
\hline & REACTOME_PATHWAY & R-HSA-3000171 & Nonintegrin membrane-ECM interactions & 14 & $6.32 E-08$ \\
\hline & REACTOME_PATHWAY & R-HSA-6785807 & Interleukin- 4 and 13 signaling & 26 & $7.33 E-08$ \\
\hline & REACTOME_PATHWAY & R-HSA-216083 & Integrin cell surface interactions & 16 & $1.25 E-07$ \\
\hline & REACTOME_PATHWAY & R-HSA-3000170 & Syndecan interactions & 9 & $1.29 E-06$ \\
\hline
\end{tabular}

Abbreviations: KEGG: Kyoto Encyclopedia of Genes and Genomes; PTC: papillary thyroid carcinoma.

JUN, DCN, BCL2, ACACB, and CXCL12), and they were chosen as the hub genes of PTC (Table 4). Furthermore, the whole PPI network was analyzed by MCODE based on the degree of importance. The top three modules were shown in Figure 5. Module 1 consists of 22 nodes and 71 edges, while Module 2 consists of 18 nodes and 54 edges, and there are 19 nodes and 54 edges in Module 3. Moreover, KEGG pathway enrichment analysis showed that genes in these three modules were predominantly associated with pathways in cancer, chemokine signaling pathway, and PI3K-Akt signaling pathway, respectively.

3.5. Validation of the DEGs by RT-PCR. To reinforce the reliability of the identified hub genes from the bioinformatics analysis, we firstly investigated the expression of these crucial genes in online website GEPIA based on TCGA and GTEx thyroid carcinoma (THCA) data. The results from TCGA and the GTEx projects containing 512 PTC samples and 337 normal samples showed that TGFB1, CXCL8, LRRK2, CD44, and CCND1 were all elevated and JUN, DCN, BCL2, $A C A C B$, and CXCL12 were reduced to some extent in PTC tissues relative to normal tissues (Figure 6(a)). Moreover, RT-PCR experiment was conducted to reconfirm these hub genes. 32 pairs of PTC tissues and their adjacent noncancerous tissues collected in our cohort were used to measure the expression of these molecules. As indicated in Figure 6(b), the results of RT-PCR showed that the mRNA level of TGFB1, CXCL8, LRRK2, CD44, and CCND1 increased by $2.44-(p=0.0066), 3.64-(p=0.0014), 6.67-(p<0.0001)$, 5.58- $(p<0.0001)$, and 3.43-fold $(p<0.0001)$ and the level of JUN, DCN, BCL2, ACACB, and CXCL12 decreased by 2.99- $(p=0.0024), 4.53-(p<0.0001), 3.57-(p<0.0035)$, 3.89- $(p<0.0001)$, and 2.37-fold $(p=0.0015)$, correspondingly. Although the fold changes varied from the data in GEO datasets and TCGA database, the total tendency of these genes from our experiment results was consistent with the databases. Besides, we evaluated the relationship between these hub genes and PTC patients' prognosis with UALCAN [26]. As shown in Figure 7, Kaplan-Meier survival curves revealed that PTC patients with high expression levels of CXCL8 $(p=0.028), D C N(p=0.017)$, BCL2 $(p=0.0045)$, and $A C A C B(p=0.0016)$ were correlated with a poor overall survival rate, suggested that these four molecules may serve as the prognostic markers for PTC. In brief, our study demonstrated that these ten hub genes may strongly link to the occurrence, progression, and prognosis of PTC and serve as a solid foundation for the future genomic individualized treatment of PTC.

\section{Discussion}

PTC is one of the most common endocrine-related cancers in the world, and the incidence increases steadily in recent decades $[4,27]$. A previous study revealed that genetic alternations such as TERT mutations, BRAF V600E mutations, and RET/PTC rearrangement all contributed to the tumor proliferation and metastasis [28]. The precise mechanism concerning the pathogenesis of PTC remains largely elusive. Therefore, it is of great importance to identify and characterize the pivotal genes associated with the development of PTC. 


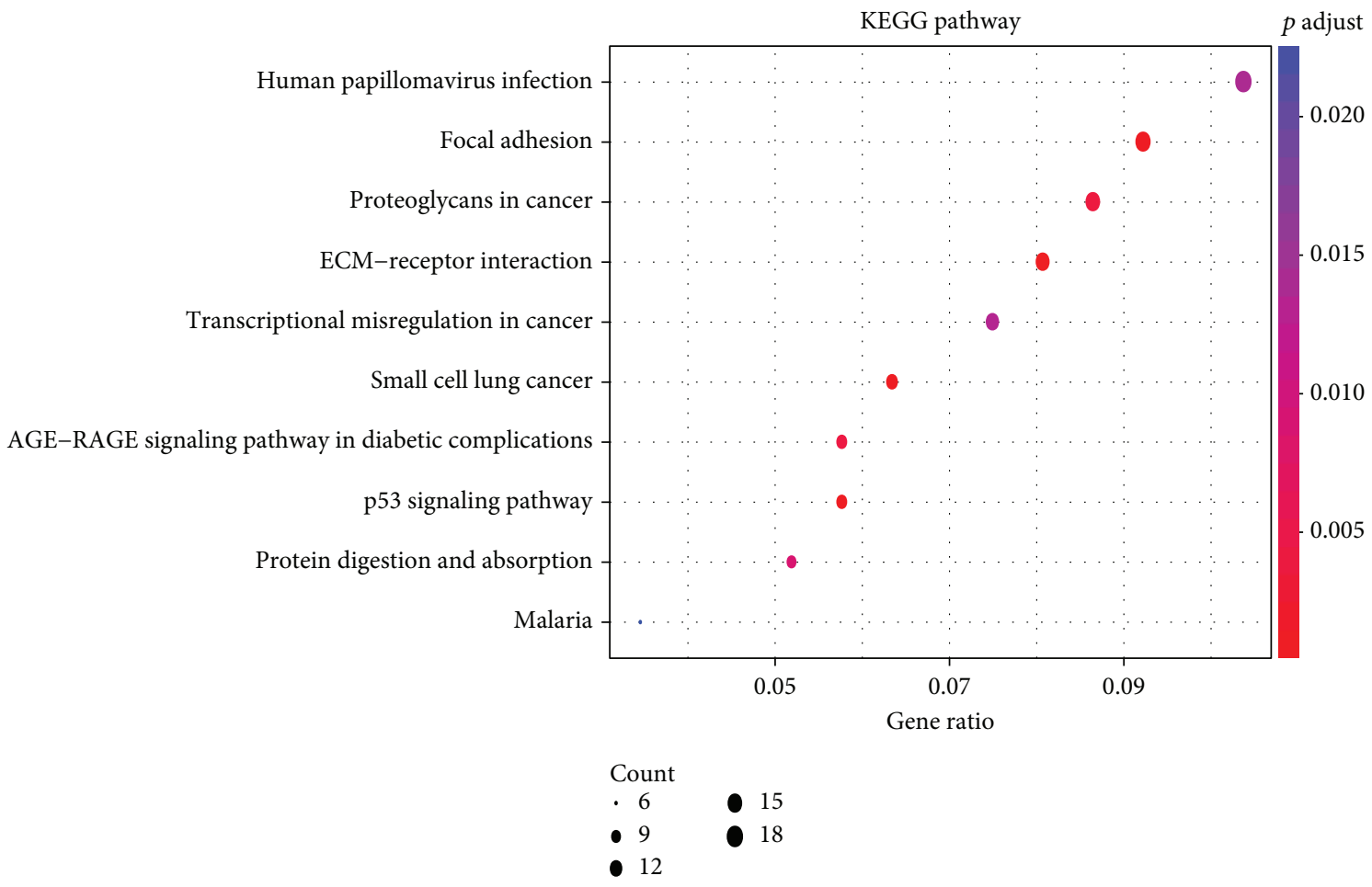

(a)

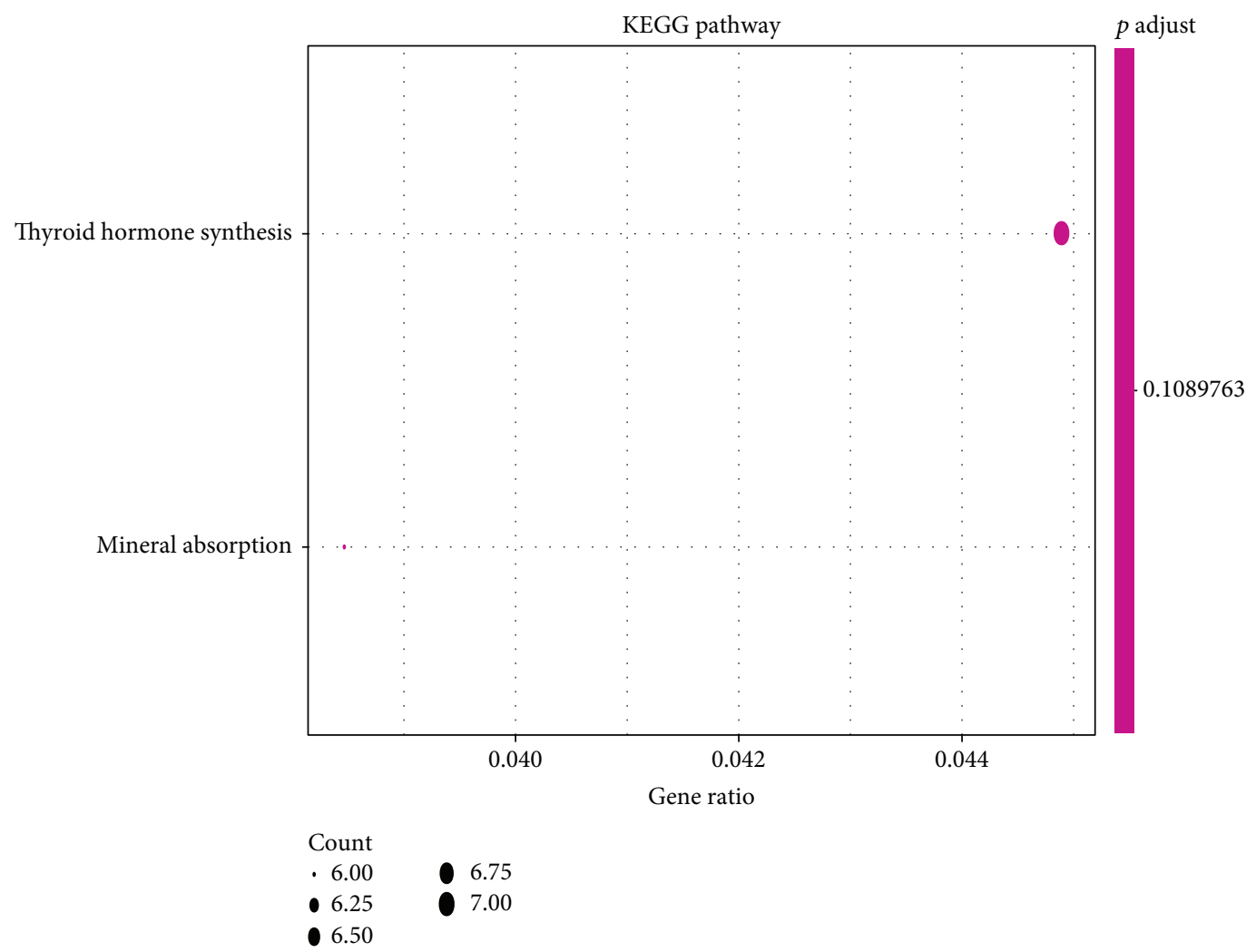

(b)

FIGURE 3: The results of KEGG pathways enrichment analysis for DEGs based on clusterProfiler: upregulated DEGs (a) and downregulated DEGs (b). Abbreviations: DEGs: differentially expressed genes; KEGG: Kyoto Encyclopedia of Genes and Genomes. 


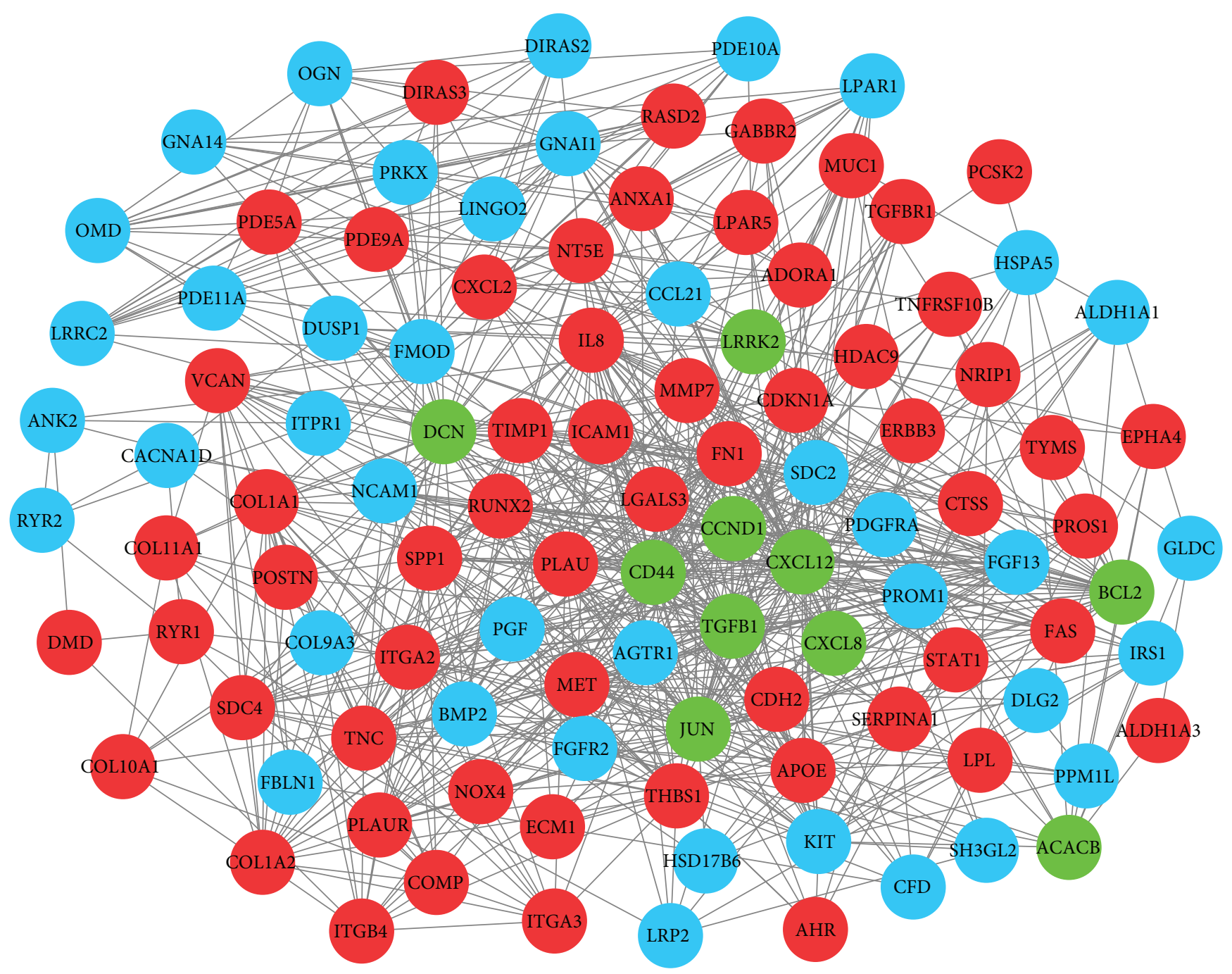

FIGURE 4: Protein-protein interaction network of differentially expressed genes. Red nodes represent upregulated genes. Blue nodes represent downregulated genes. Green nodes represent the top ten genes. Nodes $>10$ was set as cutoff criteria.

TABle 4: Top 10 genes with high node degrees in PPI network.

\begin{tabular}{|c|c|c|c|c|c|c|c|}
\hline \multirow{2}{*}{ Node } & \multirow{2}{*}{ Degree } & \multicolumn{2}{|c|}{ GSE33630 } & \multicolumn{2}{|c|}{ GSE35570 } & \multicolumn{2}{|c|}{ GSE60542 } \\
\hline & & $\log _{2} \mathrm{FC}$ & Adj. $p$ & $\log _{2} \mathrm{FC}$ & Adj. $p$ & $\log _{2} \mathrm{FC}$ & Adj. $p$ \\
\hline$T G F B 1$ & 73 & 1.62 & $3.06 \times 10^{-15}$ & 2.52 & $1.04 \times 10^{-14}$ & 1.46 & $4.23 \times 10^{-10}$ \\
\hline$J U N$ & 67 & -1.38 & $9.36 \times 10^{-11}$ & -1.55 & $1.11 \times 10^{-5}$ & -1.69 & $1.87 \times 10^{-9}$ \\
\hline CXCL8 & 62 & 2.01 & $2.22 \times 10^{-9}$ & 1.88 & $2.84 \times 10^{-7}$ & 1.11 & $1.91 \times 10^{-3}$ \\
\hline LRRK2 & 59 & 4.26 & $2.30 \times 10^{-25}$ & 5.98 & $9.14 \times 10^{-35}$ & 3.59 & $5.99 \times 10^{-14}$ \\
\hline$C D 44$ & 56 & 1.88 & $3.16 \times 10^{-15}$ & 1.54 & $7.26 \times 10^{-12}$ & 1.38 & $2.48 \times 10^{-13}$ \\
\hline$D C N$ & 47 & -1.88 & $1.58 \times 10^{-14}$ & -2.26 & $5.32 \times 10^{-12}$ & -1.75 & $8.24 \times 10^{-6}$ \\
\hline BCL2 & 46 & -1.72 & $2.79 \times 10^{-22}$ & -1.78 & $1.02 \times 10^{-27}$ & -1.88 & $6.27 \times 10^{-14}$ \\
\hline$A C A C B$ & 42 & -1.11 & $9.88 \times 10^{-13}$ & -1.56 & $3.93 \times 10^{-18}$ & -1.45 & $1.53 \times 10^{-16}$ \\
\hline CCND1 & 42 & 1.71 & $1.32 \times 10^{-20}$ & 1.51 & $5.97 \times 10^{-17}$ & 1.27 & $6.31 \times 10^{-7}$ \\
\hline CXCL12 & 40 & -1.56 & $1.43 \times 10^{-13}$ & -1.76 & $6.17 \times 10^{-10}$ & -1.74 & $1.08 \times 10^{-8}$ \\
\hline
\end{tabular}

Notes: The adj. $p$ value was calculated using Student's $t$-test. Abbreviations: PPI, protein-protein interaction. 


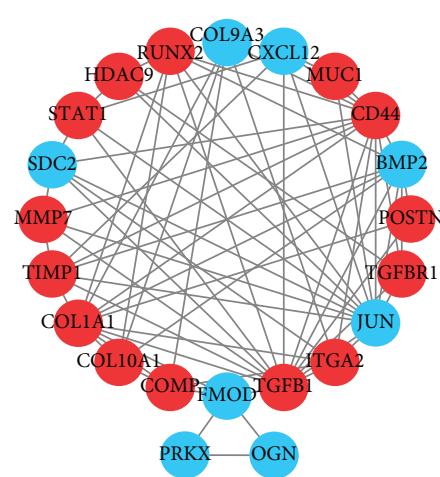

\begin{tabular}{lccccc}
\hline Expression & Category & ID & Term & Count & $p$ value \\
\hline Module 1 & KEGG_PATHWAY & hsa05200 & Pathways in cancer & 7 & $1.60 E-04$ \\
& KEGG_PATHWAY & hsa04512 & ECM-receptor interaction & 4 & $9.59 E-04$ \\
& KEGG_PATHWAY & hsa04380 & Osteoclast differentiation & 4 & 0.003113 \\
& KEGG_PATHWAY & hsa05161 & Hepatitis B & 4 & 0.004147 \\
& KEGG_PATHWAY & hsa05205 & Proteoglycans in cancer & 4 & 0.010126 \\
\hline
\end{tabular}

(a)

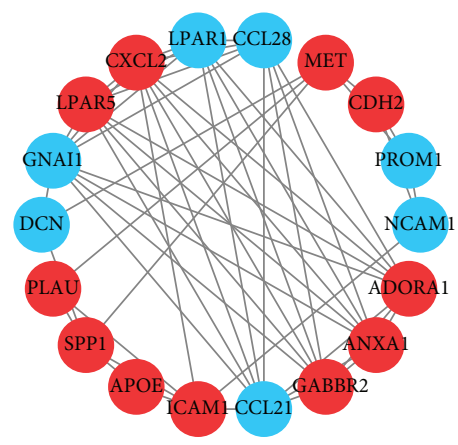

\begin{tabular}{lccccc}
\hline Expression & Category & ID & Term & Count & $p$ value \\
\hline Module 2 & KEGG_PATHWAY & hsa04026 & Chemokine signaling pathway & 4 & 0.008298 \\
& KEGG_PATHWAY & hsa04015 & Rap1 signaling pathway & 4 & 0.011565 \\
& KEGG_PATHWAY & hsa04151 & PI3K-AKT signaling pathway & 4 & 0.042587 \\
& KEGG_PATHWAY & hsa04064 & NF-kappa B signaling pathway & 3 & 0.01677 \\
& KEGG_PATHWAY & hsa05032 & Morphine addiction & 3 & 0.018258 \\
\hline
\end{tabular}

(b)

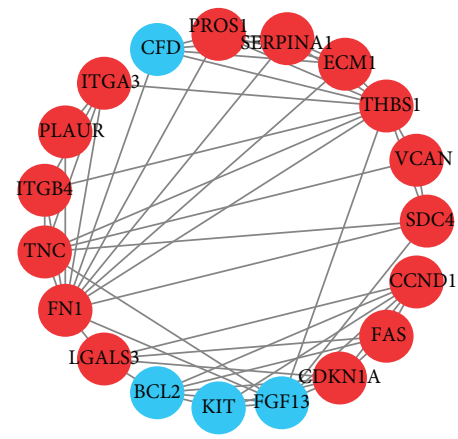

\begin{tabular}{lccccc}
\hline Expression & Category & ID & Term & Count & $p$ value \\
\hline Module 3 & KEGG_PATHWAY & hsa04151 & PI3K-AKT signaling pathway & 10 & $1.46 E-08$ \\
& KEGG_PATHWAY & hsa05200 & Pathways in cancer & 8 & $1.33 E-05$ \\
& KEGG_PATHWAY & hsa05205 & Proteoglycans in cancer & 7 & $3.43 E-06$ \\
& KEGG_PATHWAY & hsa04510 & Focal adhesion & 7 & $4.07 E-06$ \\
& KEGG_PATHWAY & hsa04512 & ECM-receptor interaction & 6 & $1.10 E-06$ \\
\hline
\end{tabular}

(c)

FIgURE 5: Top three modules with high degree of connectivity from the PPI network. (a) PPI Module 1 and KEGG pathway enrichment analysis for Module 1. (b) Module 2 and KEGG pathway analysis for Module 2. (c) Module 3 and KEGG pathway analysis for Module 3. Abbreviations: PPI: protein-protein interaction; KEGG: Kyoto Encyclopedia of Genes and Genomes.

In the current study, our results provide a comprehensive bioinformatics analysis of genes and pathways which may participated in the progression of PTC. We integrated 3 datasets including 114 PTC tissues and 126 normal tissues, which enrolled the largest number of PTC tissue samples in similar bioinformatics studies, and identified 831 DEGs (410 upregulated and 421 downregulated) at the first step [29]. The following GO analysis showed that upregulated DEGs were mainly enriched in the extracellular matrix organization, endoplasmic reticulum lumen, and glycosaminoglycan binding. In addition, downregulated DEGs were significantly involved in the organic hydroxy compound metabolic process, proteinaceous extracellular matrix, and sulfur compound binding. Subsequent KEGG pathway enrichment analysis revealed that PTC development was strongly linked to pathways, such as thyroid hormone syn- thesis, ECM-receptor interaction, and p53 signaling pathway. In addition, we constructed the PPI network for DEGs and discovered top ten hub genes (TGFB1, CXCL8, LRRK2, CD44, CCND1, JUN, DCN, BCL2, ACACB, and CXCL12) with highest degree of connectivity. Finally, the most significant 3 modules were filter from the PPI network. Corresponding genes among these modules were associated with pathways in cancer, chemokine signaling pathway, and PI3K-Akt signaling pathway. Furthermore, experimental validation is indispensable to confirm our results predicted by bioinformatics analysis. RT-PCR results verified the overexpression of TGFB1, CXCL8, LRRK2, $C D 44$, and CCND1 and the downregulation of JUN, DCN, BCL2, $A C A C B$, and CXCL12 in 32 pairs of PTC samples and their adjacent normal tissues. Among these hub genes, CXCL8, DCN, BCL2, and $A C A C B$ were identified 

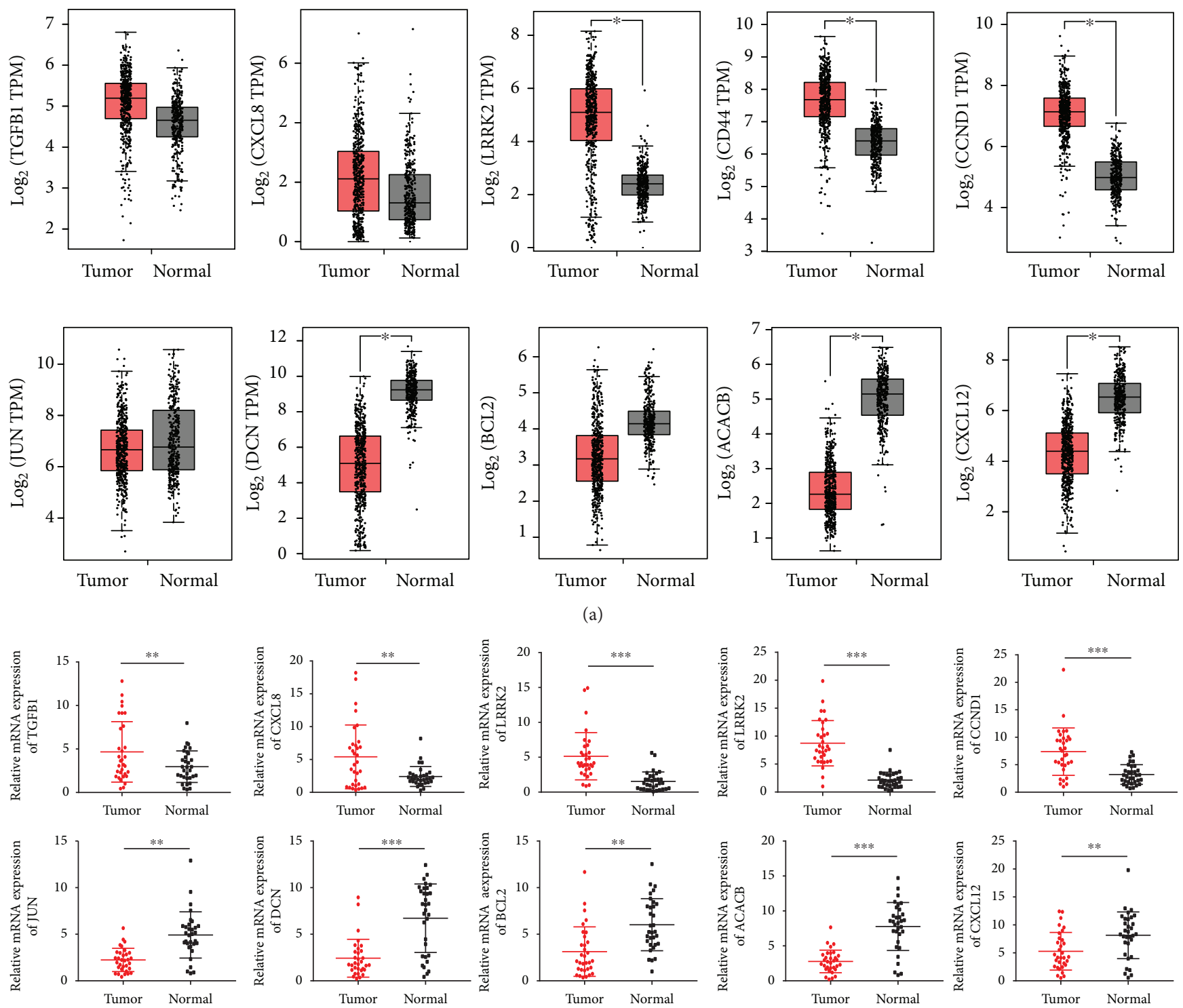

(b)

FIgURe 6: The expression level of TGFB1, CXCL8, LRRK2, CD44, CCND1, JUN, DCN, BCL2, ACACB, and CXCL12. (a) Validation of upregulated and downregulated hub genes based on TCGA and GTEx data in GEPIA. (b) Validation of the expression level of ten hub genes using RT-PCR. ${ }^{*} p<0.05,{ }^{* *} p<0.01$, and ${ }^{* * *} p<0.001$. Abbreviations: TCGA: The Cancer Genome Atlas; GTEx: Genotype-Tissue Expression; GEPIA: Gene Expression Profiling Interactive Analysis.

as clinically relevant genes using the expression status from the TCGA cohort of 504 PTC samples.

CXCL8, also named IL-8, is a member of the CXC chemokine family and produced by leukocytes and other cells. As a proinflammatory/chemoattractant cytokine, CXCL8 exerts its oncogenic effect by sustaining cell proliferation, metastasis, and angiogenesis [30]. The results of our study were in consistent with several published research papers that have reported the elevated expression of CXCL8 in PTC. Basolo et al. revealed that CXCL8 was produced by TPC-1 and TT cells with activated RET/PTC1 arrangement and its expression was regulated by multiple intracellular signaling pathways [31]. Liotti et al. reported that the CXCL8 expression level was correlated with lymph node metastases and it was overexpressed in thyrospheres and critical for self-renewal ability of thyroid cancer cells [32]. Rotondi et al. discovered that CXCL8 was highly abundant in PTCderived tumor-associated macrophages (TAMs) and promoted PTC invasion in vivo [33]. Moreover, the role of CXCL8 in thyroid cancer was recently addressed by several studies which characterized its secretion and expression; in particular, these findings were recently reviewed and CXCL8 was proposed as the chemokine playing crucial protumorigenic effects in the cancer microenvironment [34]. Due to the important position of CXCL8 in PTC, Coperchini et al. designed some experiments to test the effect of the BRAF inhibitor (PLX4720) on the basal and TNF- $\alpha$-induced CXCL8 secretions in BRAFV600E-mutated (BCPAP, 

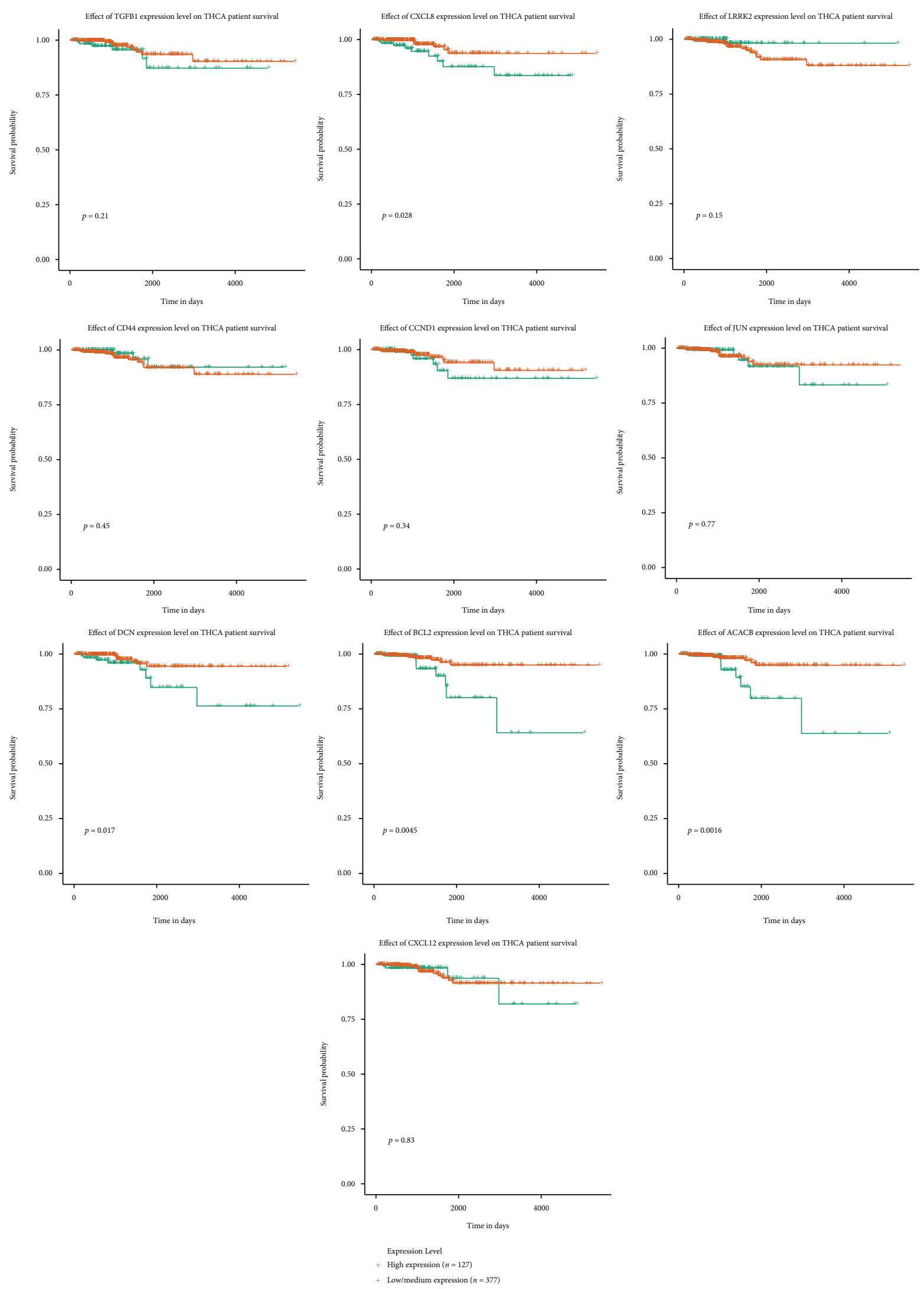

Figure 7: The Kaplan-Meier survival curve for 504 PTC patients with high and low expression of ten hub genes using data from TCGA database. Abbreviation: THCA: thyroid cancer. 
8305C, and 8505C) and RET/PTC-rearranged (TPC-1) thyroid cancer cell lines and in normal human thyrocytes (NHT). The experiment results show that PLX4720 is able to inhibit the secretion of CXCL8 in BRAFV600E-mutated thyroid cancer cells, which indicated that at least some of the antitumor activities of PLX4720 could be exerted through lowering of CXCL8 in the thyroid cancer microenvironment $[35,36]$.

Decorin $(D C N)$ was known as an extracellular protein and a pan-RTK inhibitor, which participates in cancer cell growth, spread, proinflammatory processes, and antifibrillogenesis [37]. DCN was found underexpressed in breast cancer, colon cancer, follicular thyroid cancer, and follicular variant of papillary thyroid carcinomas [38, 39]. Mechanically, DCN binds to EGFR/ErB2 extracellular functional domains and triggers mitogen-activated protein kinase activation and the increase of cytosolic $\mathrm{Ca}^{2+}$, which accelerates cell cycle arrest and induces intrinsic cell apoptosis, eventually leading to tumor cell growth inhibition [40]. Taken together, the above studies highlighted DCN as a candidate anticancer target for many types of solid tumors. In addition, we have reason to suspect that DCN have an important role in the carcinogenesis of papillary thyroid cancer.

Acetyl-CoA carboxylases (ACC), the rate-limiting enzymes involved in the de novo synthesis of fatty acids, encode two isoforms ACC1 and ACC2 (ACACB) [41]. AMPK directly inhibits ACC1 and ACC2 activity via phosphorylation of ACC1 at Ser117 and ACC2 at Ser222, resulting in the inhibition of fatty acid synthesis and accumulation of fatty acid $\beta$-oxidation $[42,43]$. A previous study has demonstrated an upregulation of ACC1 in multiple human cancers, which may promote lipogenesis to provide the energy for cancer cell growth [44]. However, we firstly found that the expression of ACC2 was at a very low level in PTC tissues and the specific mechanism of ACC2 in PTC carcinogenesis remains unclear and needs further investigation.

BCL2 is an antiapoptotic oncogene, functioning in the regulation of cell intrinsic apoptosis. There exists numerous BCL2 family members, including the antiapoptotic proteins BCL2 and BCL-xL and proapoptotic proteins Bax, Bak, Bid, Noxa, Puma, Bad, and others [45]. Once received cellular stress, the activation of these BCL2 family members releases apoptogenic proteins from the mitochondria and initiates caspase activation that results in cell apoptosis [46]. Overexpression of prosurvival BCL2 in multiple cancers, such as prostate, ovarian, and lung cancer, is a hallmark for tumorigenesis [47-49]. Nevertheless, it was significantly downregulated in colorectal cancer (CRC) and PTC [50, 51]. Wang et al. investigated the relationship between BCL2 polymorphisms and PTC susceptibility and found that BCL2 (-938 $\mathrm{C}>\mathrm{A}$ ) polymorphism showed a protective role in susceptibility to PTC [52]. Gupta et al. reported downregulated BCL2 in anaplastic papillary carcinoma (ATC) as compared to welldifferentiated thyroid cancer [53]. Besides, loss of BCL2 was associated with loss of differentiation in thyroid cancer. Our findings suggested that aberrantly expressed BCL2 may have an unknown novel function as a potential tumor suppressor in thyroid cancer progression.
Taken together, we conducted a comprehensive bioinformatics analysis of DEGs in PTC and demonstrated that ten hub genes as the biomarkers of PTC and all these molecules were validated by our RT-PCR experiments. Our findings provide novel insights into the role of potential oncogenes and tumor suppressor genes in PTC and suggest that these findings may have a great clinical significance. Understanding of these core genes in thyroid cancer development as well as the molecular mechanisms involved in this process should be consolidated in the future investigation. Meanwhile, targeting these genes, especially the clinical relevant genes (CXCL8, DCN, BCL2, and $A C A C B$ ), may provide effective therapies for the treatment of PTC. However, there is still a long way to get these genes transferred to the clinic for stratifying patients, taken as diagnostic biomarkers and even in depth for immunotherapy or for the development for oncovaccine.

\section{Data Availability}

The data used to support the findings of this study are included within the article.

\section{Conflicts of Interest}

The authors report no conflicts of interest in this work.

\section{Authors' Contributions}

Xiaoyan Li and Jing He contributed equally to this work.

\section{Acknowledgments}

This study was supported by the Science and Technology Commission of Shanghai Municipality (No. 15411952503) and National Nature Science Foundation of China (No. 81602326).

\section{References}

[1] R. L. Siegel, K. D. Miller, and A. Jemal, "Cancer statistics, 2017," CA: a Cancer Journal for Clinicians, vol. 67, no. 1, pp. 7-30, 2017.

[2] W. Chen, R. Zheng, P. D. Baade et al., "Cancer statistics in China, 2015," CA: a Cancer Journal for Clinicians, vol. 66, no. 2, pp. 115-132, 2016.

[3] C. M. Kitahara and J. A. Sosa, "The changing incidence of thyroid cancer," Nature Reviews Endocrinology, vol. 12, no. 11, pp. 646-653, 2016.

[4] C. La Vecchia, M. Malvezzi, C. Bosetti et al., "Thyroid cancer mortality and incidence: a global overview," International Journal of Cancer, vol. 136, no. 9, pp. 2187-2195, 2015.

[5] S. Leboulleux, C. Rubino, E. Baudin et al., "Prognostic factors for persistent or recurrent disease of papillary thyroid carcinoma with neck lymph node metastases and/or tumor extension beyond the thyroid capsule at initial diagnosis," The Journal of Clinical Endocrinology and Metabolism, vol. 90, no. 10, pp. 5723-5729, 2005.

[6] M. Ansari, A. A. Babaei, B. Shafiei et al., "Pathological evaluation of differentiated thyroid cancer in patients with positive serum thyroglobulin and negative iodine scan," European 
Review for Medical and Pharmacological Sciences, vol. 18, no. 13, pp. 1925-1929, 2014.

[7] M. E. Cabanillas, D. G. McFadden, and C. Durante, "Thyroid cancer," The Lancet, vol. 388, no. 10061, pp. 2783-2795, 2016.

[8] J. F. Carrillo, M. Friasmendivil, F. J. Ochoa-Carrillo, and M. Ibarra, "Accuracy of fine-needle aspiration biopsy of the thyroid combined with an evaluation of clinical and radiologic factors," Otolaryngology - Head and Neck Surgery, vol. 122, no. 6, pp. 917-921, 2000.

[9] C. Are and A. R. Shaha, "Anaplastic thyroid carcinoma: biology, pathogenesis, prognostic factors, and treatment approaches," Annals of Surgical Oncology, vol. 13, no. 4, pp. 453-464, 2006.

[10] Y. Cohen, M. Xing, E. Mambo et al., "BRAF mutation in papillary thyroid carcinoma," Journal of the National Cancer Institute, vol. 95, no. 8, pp. 625-627, 2003.

[11] P. Soares, V. Trovisco, A. S. Rocha et al., "BRAF mutations and RET/PTC rearrangements are alternative events in the etiopathogenesis of PTC," Oncogene, vol. 22, no. 29, pp. 45784580, 2003.

[12] T. Fukushima, S. Suzuki, M. Mashiko et al., "BRAF mutations in papillary carcinomas of the thyroid," Oncogene, vol. 22, no. 41, pp. 6455-6457, 2003.

[13] D. Zhou, Z. Li, and X. Bai, "BRAFV600E and RET/PTC promote proliferation and migration of papillary thyroid carcinoma cells in vitro by regulating nuclear factor- $\kappa \mathrm{B}$," Medical Science Monitor, vol. 23, pp. 5321-5329, 2017.

[14] J. A. Knauf, H. Kuroda, S. Basu, and J. A. Fagin, "RET/PTCinduced dedifferentiation of thyroid cells is mediated through Y1062 signaling through SHC-RAS-MAP kinase," Oncogene, vol. 22, no. 28, pp. 4406-4412, 2003.

[15] R. J. Neely, M. S. Brose, C. M. Gray et al., "The RET/PTC3 oncogene activates classical NF- $\kappa$ B by stabilizing NIK," Oncogene, vol. 30, no. 1, pp. 87-96, 2011.

[16] A. Y. M. Au, C. McBride, K. G. Wilhelm Jr. et al., "PAX8-peroxisome proliferator-activated receptor $\gamma(\operatorname{PPAR} \gamma)$ disrupts normal PAX8 or PPAR $\gamma$ transcriptional function and stimulates follicular thyroid cell growth," Endocrinology, vol. 147, no. 1, pp. 367-376, 2006.

[17] L. Lacroix, V. Lazar, S. Michiels et al., "Follicular thyroid tumors with the PAX8-PPARgamma1 rearrangement display characteristic genetic alterations," The American Journal of Pathology, vol. 167, no. 1, pp. 223-231, 2005.

[18] W. O. Lui, T. Foukakis, J. Lidén et al., "Expression profiling reveals a distinct transcription signature in follicular thyroid carcinomas with a PAX8-PPAR $\gamma$ fusion oncogene," Oncogene, vol. 24, no. 8, pp. 1467-1476, 2005.

[19] J. Di Cristofaro, M. Silvy, A. Lanteaume, M. Marcy, P. Carayon, and C. De Micco, "Expression of tpo mRNA in thyroid tumors: quantitative PCR analysis and correlation with alterations of ret, Braf, ras and pax8 genes," EndocrineRelated Cancer, vol. 13, no. 2, pp. 485-495, 2006.

[20] W. Liang and F. Sun, "Identification of key genes of papillary thyroid cancer using integrated bioinformatics analysis," Journal of Endocrinological Investigation, vol. 41, no. 10, pp. 12371245, 2018.

[21] S. Davis and P. S. Meltzer, "GEOquery: a bridge between the Gene Expression Omnibus (GEO) and BioConductor," Bioinformatics, vol. 23, no. 14, pp. 1846-1847, 2007.

[22] Z. Tang, C. Li, B. Kang, G. Gao, C. Li, and Z. Zhang, "GEPIA: a web server for cancer and normal gene expression profiling and interactive analyses," Nucleic Acids Research, vol. 45, no. W1, pp. W98-W102, 2017.

[23] G. Yu, L. G. Wang, Y. Han, and Q. Y. He, “clusterProfiler: an R package for comparing biological themes among gene clusters," OMICS, vol. 16, no. 5, pp. 284-287, 2012.

[24] F. He, P. Lv, X. Zhao et al., "Predictive value of circulating miR-328 and miR-134 for acute myocardial infarction," Molecular and Cellular Biochemistry, vol. 394, no. 1-2, pp. 137-144, 2014.

[25] F. C. He, W. W. Meng, Y. H. Qu et al., "Expression of circulating microRNA-20a and let-7a in esophageal squamous cell carcinoma," World Journal of Gastroenterology, vol. 21, no. 15, pp. 4660-4665, 2015.

[26] D. S. Chandrashekar, B. Bashel, S. A. H. Balasubramanya et al., "UALCAN: a portal for facilitating tumor subgroup gene expression and survival analyses," Neoplasia, vol. 19, no. 8, pp. 649-658, 2017.

[27] J. He, Y. Jin, M. Zhou et al., "Solute carrier family 35 member F2 is indispensable for papillary thyroid carcinoma progression through activation of transforming growth factor- $\beta$ type I receptor/apoptosis signal-regulating kinase $1 /$ mitogen-activated protein kinase signaling axis," Cancer Science, vol. 109, no. 3, pp. 642-655, 2018.

[28] M. Xing, "Molecular pathogenesis and mechanisms of thyroid cancer," Nature Reviews Cancer, vol. 13, no. 3, pp. 184-199, 2013.

[29] Y. Zhao, X. Liu, L. Zhong et al., "The combined use of miRNAs and mRNAs as biomarkers for the diagnosis of papillary thyroid carcinoma," International Journal of Molecular Medicine, vol. 36, no. 4, pp. 1097-1103, 2015.

[30] M. Rotondi, F. Coperchini, and L. Chiovato, "CXCL8 in thyroid disease: from basic notions to potential applications in clinical practice," Cytokine \& Growth Factor Reviews, vol. 24, no. 6, pp. 539-546, 2013.

[31] F. Basolo, R. Giannini, A. Toniolo et al., "Establishment of a non-tumorigenic papillary thyroid cell line (FB-2) carrying the RET/PTC1 rearrangement," International Journal of Cancer, vol. 97, no. 5, pp. 608-614, 2002.

[32] F. Liotti, F. Collina, E. Pone et al., "Interleukin-8, but not the related chemokine CXCL1, sustains an autocrine circuit necessary for the properties and functions of thyroid cancer stem cells," Stem Cells, vol. 35, no. 1, pp. 135-146, 2017.

[33] M. Rotondi, F. Coperchini, F. Latrofa, and L. Chiovato, "Role of chemokines in thyroid cancer microenvironment: is CXCL8 the main player?," Frontiers in Endocrinology, vol. 9, p. 314, 2018.

[34] F. Coperchini, L. Croce, M. Denegri et al., "The BRAFinhibitor PLX4720 inhibits CXCL8 secretion in BRAFV600E mutated and normal thyroid cells: a further anti-cancer effect of BRAF-inhibitors," Scientific Reports, vol. 9, no. 1, article 4390, 2019.

[35] O. Awwad, F. Coperchini, P. Pignatti et al., "The AMPKactivator AICAR in thyroid cancer: effects on CXCL8 secretion and on CXCL8-induced neoplastic cell migration," Journal of Endocrinological Investigation, vol. 41, no. 11, pp. 1275-1282, 2018.

[36] W. Fang, L. Ye, L. Shen et al., “Tumor-associated macrophages promote the metastatic potential of thyroid papillary cancer by releasing CXCL8," Carcinogenesis, vol. 35, no. 8, pp. 17801787, 2014.

[37] S. Goldoni and R. V. Iozzo, "Tumor microenvironment: modulation by decorin and related molecules harboring 
leucine-rich tandem motifs," International Journal of Cancer, vol. 123, no. 11, pp. 2473-2479, 2008.

[38] G. Li, M. Li, X. Liang et al., "Identifying DCN and HSPD1 as potential biomarkers in colon cancer using 2D-LC-MS/MS combined with iTRAQ technology," Journal of Cancer, vol. 8, no. 3, pp. 479-489, 2017.

[39] L. A. T. Arnaldi, R. C. Borra, R. M. B. Maciel, and J. M. Cerutti, "Gene expression profiles reveal that DCN, DIO1, and DIO2 are underexpressed in benign and malignant thyroid tumors," Thyroid, vol. 15, no. 3, pp. 210-221, 2005.

[40] W. Zhang, Y. Ge, Q. Cheng, Q. Zhang, L. Fang, and J. Zheng, "Decorin is a pivotal effector in the extracellular matrix and tumour microenvironment," Oncotarget, vol. 9, no. 4, pp. 5480-5491, 2018.

[41] C. Wang, S. Rajput, K. Watabe, D. F. Liao, and D. Cao, "Acetyl-CoA carboxylase-a as a novel target for cancer therapy," Frontiers in Bioscience, vol. 2, pp. 515-526, 2010.

[42] R. U. Svensson, S. J. Parker, L. J. Eichner et al., "Inhibition of acetyl-CoA carboxylase suppresses fatty acid synthesis and tumor growth of non-small-cell lung cancer in preclinical models," Nature Medicine, vol. 22, no. 10, pp. 1108-1119, 2016.

[43] G. R. Steinberg and B. E. Kemp, "AMPK in health and disease,” Physiological Reviews, vol. 89, no. 3, pp. 1025-1078, 2009.

[44] J. V. Swinnen, K. Brusselmans, and G. Verhoeven, "Increased lipogenesis in cancer cells: new players, novel targets," Current Opinion in Clinical Nutrition and Metabolic Care, vol. 9, no. 4, pp. 358-365, 2006.

[45] A. Ruefli-Brasse and J. C. Reed, "Therapeutics targeting Bcl-2 in hematological malignancies," The Biochemical Journal, vol. 474, no. 21, pp. 3643-3657, 2017.

[46] Z. Jin and W. S. El-Deiry, "Overview of cell death signaling pathways," Cancer Biology \& Therapy, vol. 4, no. 2, pp. 139163, 2005.

[47] S. D. Catz and J. L. Johnson, "BCL-2 in prostate cancer: a minireview," Apoptosis, vol. 8, no. 1, pp. 29-37, 2003.

[48] N. S. Anderson, L. Turner, S. Livingston, R. Chen, S. V. Nicosia, and P. A. Kruk, "Bcl-2 expression is altered with ovarian tumor progression: an immunohistochemical evaluation," $J$ Ovarian Res., vol. 2, no. 1, p. 16, 2009.

[49] V. K. Anagnostou, F. J. Lowery, V. Zolota et al., "High expression of BCL-2 predicts favorable outcome in non-small cell lung cancer patients with non squamous histology," $B M C$ Cancer, vol. 10, no. 1, p. 186, 2010.

[50] S. Wu, F. Wu, and Z. Jiang, "Identification of hub genes, key miRNAs and potential molecular mechanisms of colorectal cancer," Oncology Reports, vol. 38, no. 4, pp. 2043-2050, 2017.

[51] T. G. Radu, L. Mogoanta, C. J. Busuioc, C. Stanescu, and F. Grosu, "Histological and immunohistochemical aspects of papillary thyroid cancer," Romanian Journal of Morphology and Embryology, vol. 56, 2 Supplement, pp. 789-795, 2015.

[52] Y. X. Wang, L. Zhao, X. Y. Wang, C. M. Liu, and S. G. Yu, "Role of Caspase 8, Caspase 9 and Bcl-2 polymorphisms in papillary thyroid carcinoma risk in Han Chinese population," Medical Oncology, vol. 29, no. 4, pp. 2445-2451, 2012.

[53] A. Gupta, S. Jain, N. Khurana, and A. K. Kakar, "Expression of p63 and Bcl-2 in malignant thyroid tumors and their correlation with other diagnostic immunocytochemical markers," Journal of Clinical and Diagnostic Research, vol. 10, no. 7, pp. EC04-EC08, 2016. 


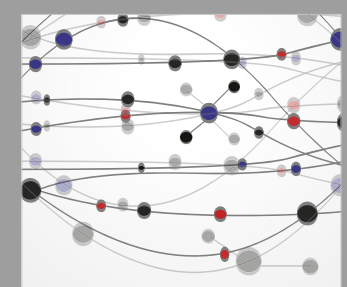

The Scientific World Journal
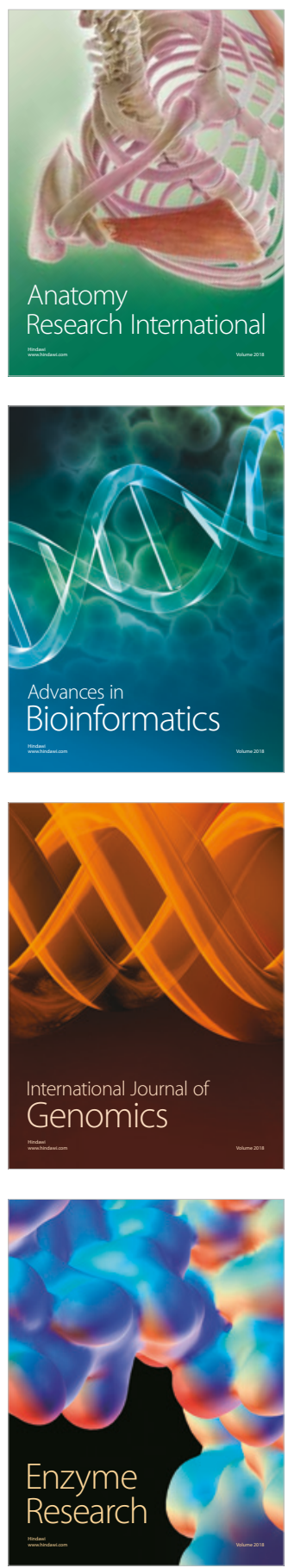
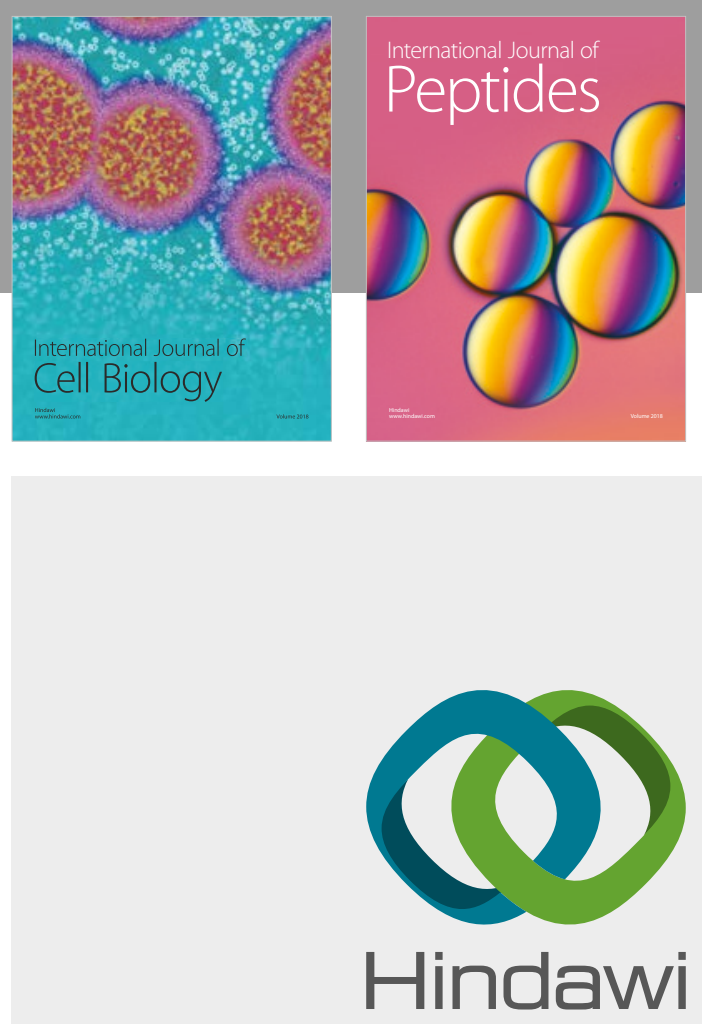

Submit your manuscripts at

www.hindawi.com
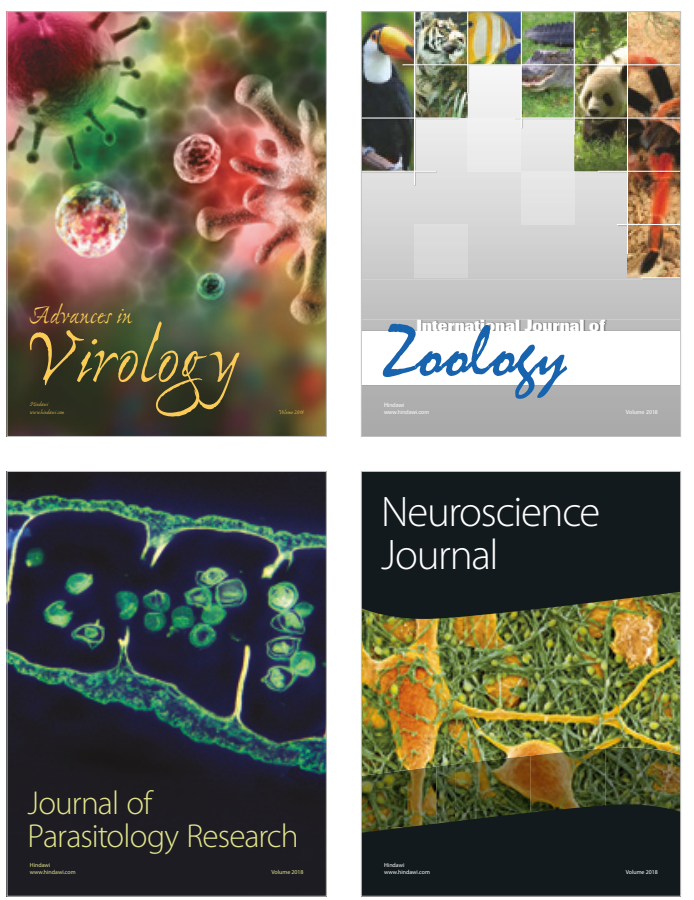
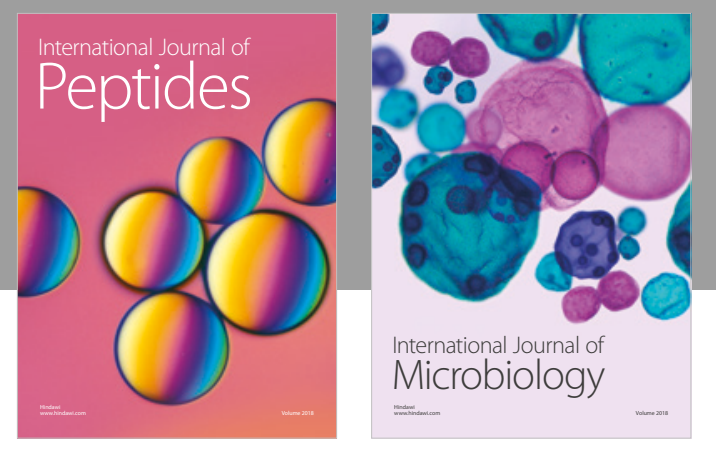

nternational Journal of Microbiology
Journal of
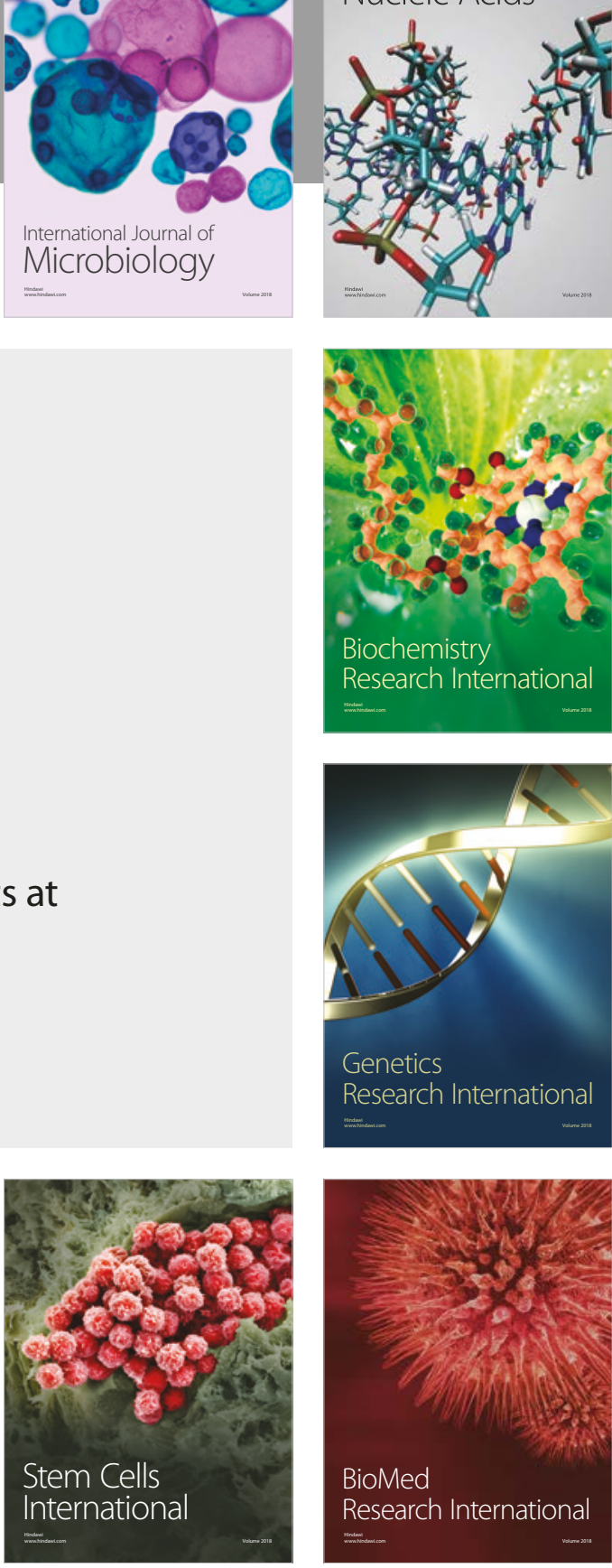
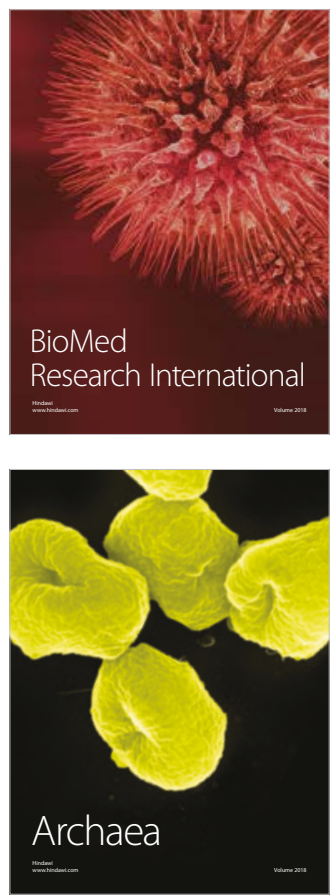